\title{
The Parker Model: Applying a Qualitative Three-Step Approach to Optimally Utilize Input from Stakeholders When Introducing New Device Technologies in the Management of Chronic Rheumatic Diseases
}

\author{
Tanja S. Jørgensen $^{1}$ (i) $\cdot$ Marie Skougaard $^{1} \cdot$ Peter C. Taylor $^{2} \cdot$ Hans C. Asmussen $^{3}$ (D) \\ Anne Lee ${ }^{4}$ (D) - Louise Klokker ${ }^{1}$ (D) - Louise Svejstrup ${ }^{5}$ Irina Mountian ${ }^{6}$. \\ Henrik Gudbergsen $^{1}$ • Lars Erik Kristensen ${ }^{1}$
}

Published online: 11 June 2018

(C) The Author(s) 2018

\begin{abstract}
Background and Objective Qualitative methods such as semi-structured interviews and focus-groups are used to evaluate the applicability and relevance of device technologies in clinical practice, but when used alone, often lack generalizability. This study aimed to assess the face validity and feasibility of using a composite, three-step qualitative method (the Parker Model), to inform the development and implementation of ava ${ }^{\circledR}$, an electromechanical device (e-Device) for subcutaneous self-
\end{abstract}

Electronic supplementary material The online version of this article (https://doi.org/10.1007/s40271-018-0306-8) contains supplementary material, which is available to authorized users.

Lars Erik Kristensen

lars.erik.kristensen@regionh.dk

1 The Parker Institute, Copenhagen University Hospital, Bispebjerg, Frederiksberg, Denmark

2 Nuffield Department of Orthopaedics, Rheumatology and Musculoskeletal, Sciences, Kennedy Institute of Rheumatology, University of Oxford, Oxford, UK

3 NATiON, 110 A, Refshalevej, Room 205, 1432 Copenhagen, Denmark

4 University of Southern Denmark, J.B. Winsløws Vej 9B, 5000 Odense, Denmark

5 Department of Rheumatology, Copenhagen University Hospital, Bispebjerg, Frederiksberg, Denmark

6 UCB Pharma, Allée de la Recherche, 60, 1070 Brussels, Belgium administration of the biologic, certolizumab pegol (CZP), used to treat rheumatic diseases.

Methods The Parker Model combines concept mapping (CM), participatory design (PD), and stakeholder evaluation (SE). CM, a structured group process, was used to identify patients' opinions and concerns regarding the e-Device. Patients used this information in iterative PD sessions to create personal e-Device prototypes in cooperation with a designer and a healthcare professional. SE was performed based on semi-structured group and individual interviews with patients and disease-management stakeholders.

Results The study recruited 14 patients, two doctors, two nurses, one medical secretary, and four other public servants. Three CM workshops revealed four key considerations: technical usability, physical design, concerns, and enthusiasm. Four personalized prototypes were developed during PD sessions. SE confirmed that the identified considerations were pivotal for the implementation and adaptation of the e-Device.

Conclusions This study is the first to apply a composite, qualitative research model when introducing an e-Device for the treatment and management of rheumatic disease. Results show that input from patients and other stakeholders using the Parker Model can add value to the development and implementation of an e-Device. 


\section{Key Points for Decision Makers}

The Parker Model is a composite, qualitative research model designed to evaluate the development and implementation of new medical device technologies.

The Parker Model combines three distinct methodologies (concept mapping, participatory design, and stakeholder evaluation) which support the flow of information between participants, helping to elucidate key themes influencing user responses to new device technologies.

This study used a new electromechanical selfinjection device (e-Device) to face validate the Parker Model. Feedback from patients and other key stakeholders demonstrated that the model comprehensively captured all constraining concepts related to the device's design and use, generated feasible solutions to overcome these constraints, and was associated with patients reporting high levels of empowerment.

\section{Introduction}

Biologic agents, such as tumor necrosis factor- $\alpha$ inhibitors (anti-TNFs), have emerged as important, effective therapeutics for many chronic inflammatory conditions, including rheumatoid arthritis (RA), psoriatic arthritis (PsA) and ankylosing spondylitis (AS) [1-3]. The efficacy and safety of anti-TNF therapeutics are the principal factors affecting both patients' experience and treatment outcomes. However, other treatment-related characteristics also influence patient satisfaction and their compliance and persistence (adherence) to treatment [4]. For example, biologic agents are often self-injected, but this can be difficult for patients with rheumatic disease, which is often associated with reduced dexterity $[5,6]$.

Adherence to therapy is suboptimal across multiple chronic conditions including rheumatic diseases [4], creating a significant barrier to achieving therapeutic outcomes [7]. The introduction of new technologies for the management of chronic conditions, which effectively meet patients' needs, may advance patient engagement and empowerment, and improve adherence to long-term treatment [8]. To achieve this, end-user perspectives need to be considered during device development and implementation. Currently, widely diverging methods are used to assess end-user perspectives, including combinations of focus groups, semi-structured interviews, and ethnographic studies [9]. Standardizing the methods used to obtain end user feedback on the use and design of new medical devices would help ensure consistent and effective enduser involvement in the development and implementation of medical devices.

The objective of the current study was to evaluate the face validity and feasibility of the Parker Model-a novel, composite, qualitative research approach —as a method of assessing end-user perspectives on new self-injection device technologies. The Parker Model is a three-step, synergistic process comprising concept mapping (CM), participatory design (PD), and stakeholder evaluation (SE) which explores the applicability and relevance of introducing the new technology in real-life clinical practice. Here, we describe and evaluate the use of the Parker Model in the implementation of $\mathrm{ava}^{\circledR}$, a reusable, electronic device (e-Device) for self-injecting single-use, disposable, pre-filled dose-dispenser cartridges of certolizumab pegol (CZP) [an anti-TNF approved for the treatment of RA, PsA, and axial spondyloarthritis (axSpA)] [10]. CM was used to identify the core concepts underlying patients' thoughts on the e-Device design and functionality. PD took the insights gained during the $\mathrm{CM}$ and investigated how the device could be further adapted to meet individual patient needs. SE was used to gather insights from patients and key disease-management stakeholders to inform the implementation and future development of the e-Device.

\section{Methods}

\subsection{Patients and Eligibility}

Patients receiving CZP treatment were recruited from the Department of Rheumatology, Copenhagen University Hospital, Bispebjerg and Frederiksberg, Denmark. To be eligible to participate, patients had to be aged 18-85 years, diagnosed with moderate to severe RA, or spondyloarthritis (SpA) (split into PsA and AS). Patients were consecutively recruited from the outpatient clinic to participate in the Parker Model until qualitative data saturation (defined as the presence of redundancy in emerging concepts) was achieved. If data saturation was not achieved after the first three CM sessions were run, two additional sessions would be run.

Prior to the first CM session, all participants attended a meeting where they were provided with the e-Device, instructions for use and a dummy dose-dispenser cartridge to familiarize themselves with the design (Fig. 1). To ensure each participant understood the design features of the e-Device, a trained instructor explained how the e-Device would be used by patients. No simulated injections were performed, but the patients were allowed to 
Fig. 1 The ava ${ }^{\circledR}$ e-Device: contents of the ava ${ }^{\circledR}$ kit (a); key features of ava ${ }^{\circledR}(\mathbf{b})$. The ava ${ }^{\circledR}$ kit comes in a storage case with the ava ${ }^{\circledR}$ device, a user manual, a welcome booklet giving an introduction to and overview of ava ${ }^{\circledR}$, a help line contact card to assist patients with any questions or concerns they may have about ava ${ }^{\circledR}$, an electrical charger (including regional plug), 2 spare needle caps (green), a USB/micro USB cable, and a reusable dosedispenser cartridge that does not contain a needle, syringe or any medication, which allows patients to practice and explore the injection process. $e$-Device electromechanical device (a)

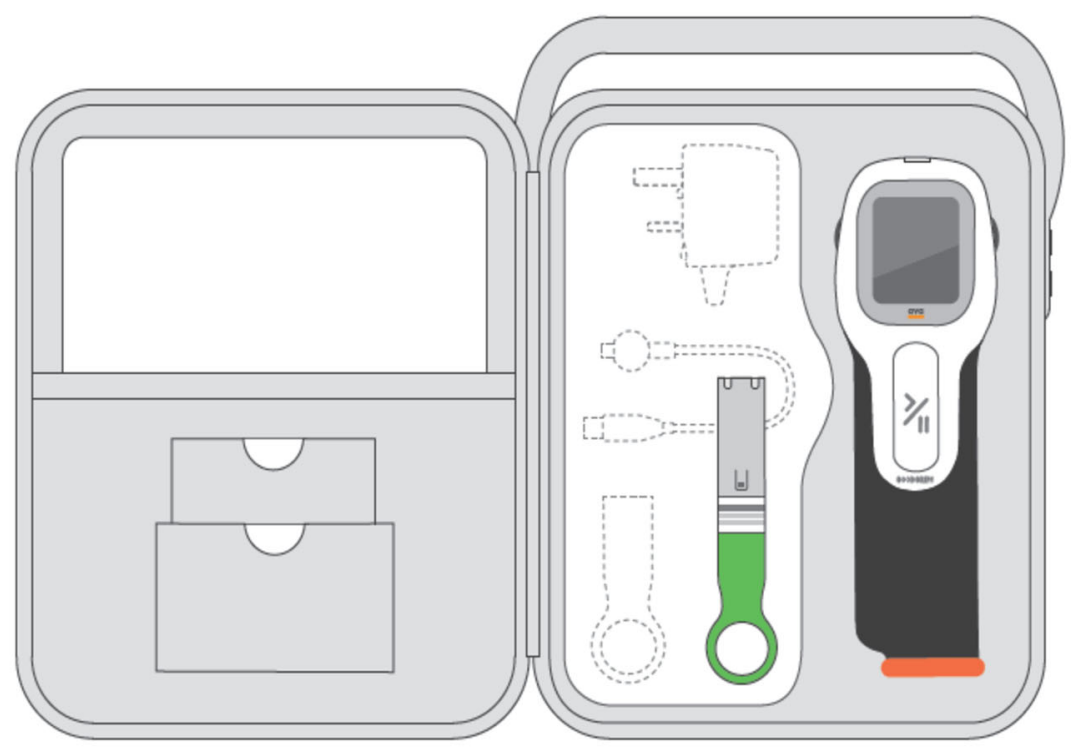

(b)

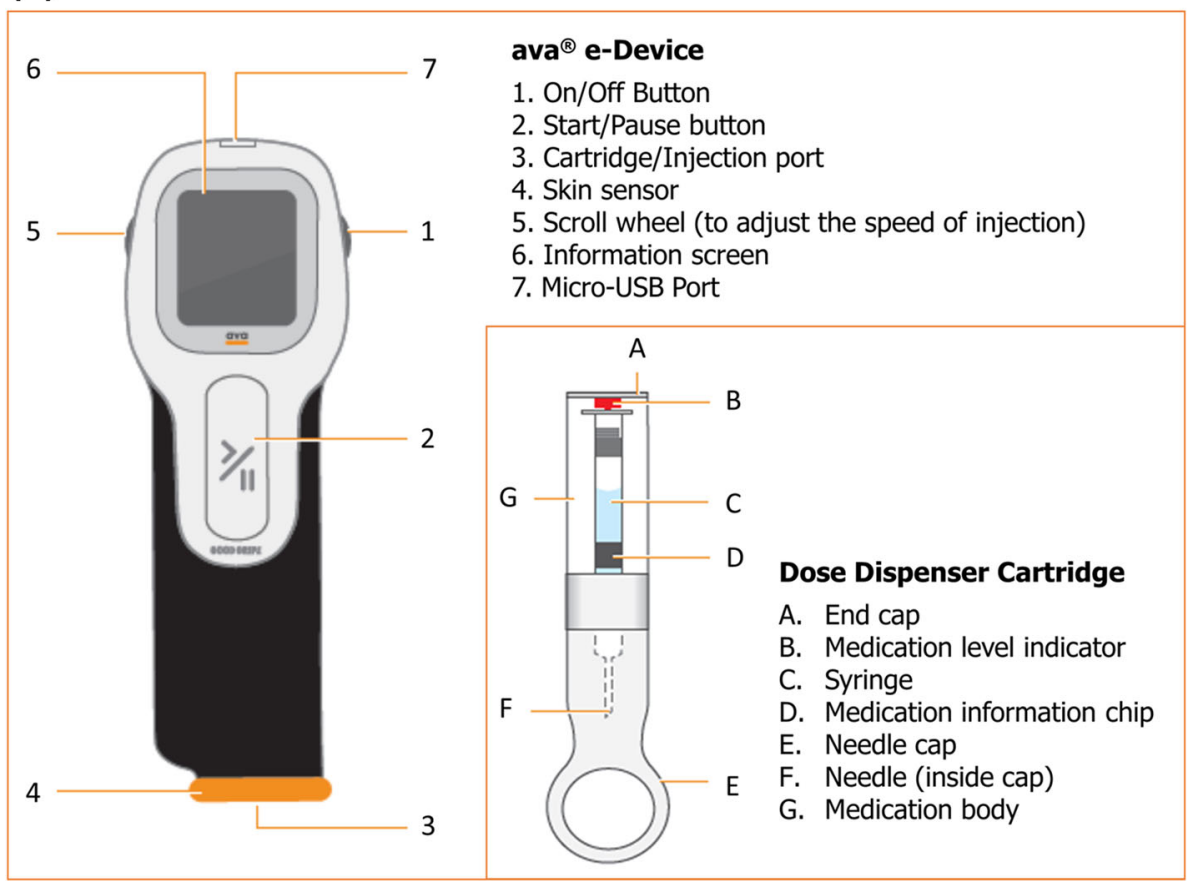

practice and explore the injection process with the e-Device using a reusable dose-dispenser cartridge that did not contain a needle, syringe or any medication.

All procedures were performed in accordance with the ethical standards of the responsible committee on human experimentation (institutional and national) and with the Helsinki Declaration of 1964, as revised in 2013. Informed consent was obtained from all patients before being included in the study, and the project was approved by the Regional Ethical Committee (file number: 16016608).

\subsection{The Parker Model}

The Parker model was developed and conducted at the Parker Institute, Copenhagen F, Denmark between November 2016 and January 2017 and comprises three qualitative methods: CM, PD, and SE (Fig. 2). The methods were selected so that information could be easily transferred between each component of the study, ensuring no insights were lost. 


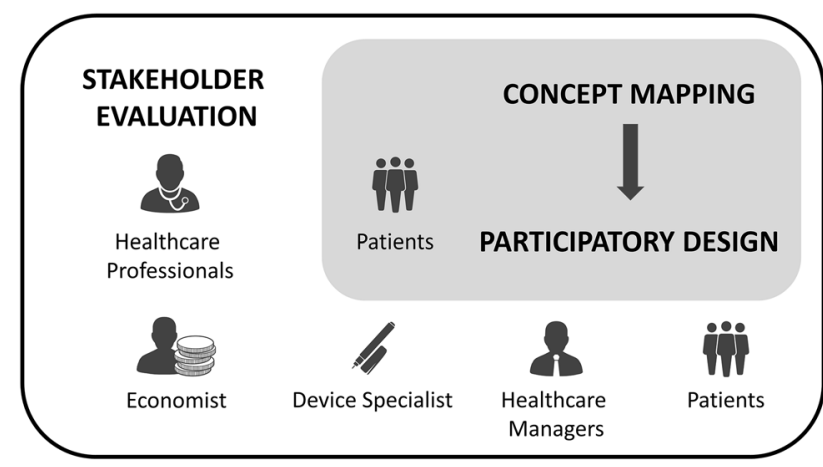

Fig. 2 The Parker Model used to gain stakeholder input on the design and implementation of ava ${ }^{\circledR}$. Concept mapping and participatory design were used to gather the patients' perception of the relevance of the e-Device, and stakeholder evaluation provided a broader perspective of both the relevance and the implementation of the e-Device through the engagement of healthcare professionals, healthcare managers, a device specialist and an economist, in addition to patients. $e$-Device electromechanical device

The CM involved full-day, patient group workshops to capture the main concepts underpinning patients' thoughts regarding a hypothetical switch from CZP treatment by self-injection using a pre-filled syringe (PFS) to self-administration using an e-Device. The concepts identified in the $\mathrm{CM}$ workshops were used in three iterative, full-day PD sessions to develop personalized prototypes of an e-Device that patients felt was 'ideal' and met their individual needs. Finally, SE was conducted via interviews with patients who were unable to participate for the full-day $\mathrm{CM}$ and $\mathrm{PD}$ workshops, and key disease-management stakeholders, to explore the applicability and relevance of implementing the e-Device.

\subsubsection{Concept Mapping}

$\mathrm{CM}$ is a formal group process with a structured approach used to identify and organize ideas on a topic of interest. It is highly effective for the development of outcome measures, such as key patient considerations, when using a device for the first time [11]. In this study, CM was conducted through three, full-day, focus groups with patients with RA or SpA. At the start of each focus group the CM process was introduced and directed as detailed in Supplementary Figure 1 [see the Electronic Supplementary Material (ESM)]. For more detailed information on the CM methodology please refer to the 'Supplementary Methods.'

Clustering analysis was performed on the participant statements generated during the three focus group CM workshops using multidimensional scaling (MDS) analysis (CS Global MAX; Concept Systems, Inc.) [12]. The $x$ and $y$ values from the MDS were used to perform a hierarchical cluster analysis which divided the statements into nonoverlapping clusters [13, 14]; any duplicate statements within the concept maps were removed $[11,15]$. Independent and thematic analysis of the reduced statement pool were performed separately by two authors (L. Klokker and T.S. Jørgensen), to identify common clusters while preserving both the exact wording of the statements and the cluster labels assigned to them by patients during the workshop (described in Supplementary Figure 1).

To identify which issues were most important for patients with rheumatic disease treated with biologics, patients were asked to rate the importance of each statement on a 5-point scale, from 1 ('not important' for people with RA or PsA receiving biologics) to 5 ('very important'). Mean and median ratings of importance assigned by the patients for each statement were calculated using Wilcoxon two-sample test.

\subsubsection{Participatory Design}

Patients who participated in the CM workshops and selfinjected CZP using a PFS were invited to participate in three iterative PD sessions to investigate how the device could be further adapted to meet individual patient needs. Patients were asked to use the PD sessions to 'develop an ideal device' based on their specific individual needs and the concepts identified in the CM workshops. Each PD session took the form of a non-structured interview of 70 min duration. A healthcare professional (HCP) (T.S. Jørgensen) and a product designer (H.C. Asmussen) were present at all sessions to act as consultants to the patients.

At the beginning of each PD session the designer presented the current state of development; for session 1, this was the original e-Device. At the end of each PD workshop, the patient and designer agreed to a set of key findings identified during the session. Using these findings and any patient sketches, the device designer then produced a device prototype using paper and clay. The prototype was presented to the patient at the beginning of the consecutive session for their feedback, which was then used to iteratively improve the subsequent version of the prototype. At the end of the third session, the patient was presented with a 3D rendered image of the final version of their prototype. A total of four prototypes (one for each participant) were produced.

\subsubsection{Stakeholder Evaluation}

SE evaluated the applicability and relevance of introducing the e-Device, using a combination of individual and group interviews with key disease-management stakeholders and patients. Disease management stakeholders from within the Department of Rheumatology, the Regional Clinical Pharmacological Department, and the Regional Service Center for Research and Innovation were recruited by 
email invitation. Patients eligible for the study who were unable to participate in the full-day CM workshops were recruited for the $\mathrm{SE}$.

Topics of discussion in the SE interviews included drivers and barriers regarding the standard route of injection (PFS) versus the e-Device, and how various elements of the e-Device could be adapted or improved in the next generation of the e-Device to better meet patients' needs.

The interviews were guided and analyzed using theorybased SE, which utilized three theories [16]. Situation theory was used to examine how stakeholders perceived the design features of the e-Device compared with the PFS, understand how the e-Device would be implemented in clinical practice, and identify any perceived challenges with the device's implementation. Normative theory was used to evaluate stakeholders' values, ideals, and goals with regard to introducing and implementing the e-Device. Causal theory was used to examine stakeholder's opinions on how an e-Device would directly or indirectly impact (either positively or negatively) current challenges and values associated with the PFS.

A transcript of each interview was produced. The transcripts were assessed to identify any comments related to current practice, ideals for practice, and how the e-Device might impact on these issues. Statements in each interview transcript were grouped into themes and coded accordingly $[17,18]$. The sections of the transcript text marked with the same theme code across all interviews were compiled and read with the aim of interpreting the meaning of, and identifying any differences between, stakeholder statements.

\subsection{Patient-Reported Outcome Measures}

To support the face validation and feasibility of the Parker Model as well as investigate the impact of being part of the project, following completion of the workshops, patients were asked to complete a questionnaire that included three validated instruments, translated into Danish: the Health Education Impact Questionnaire (heiQ), to evaluate patient education and empowerment [19-22]; the Service User Technology Acceptability Questionnaire (SUTAQ), to assess the acceptability of technology in general [23]; and the e-Health Literacy Questionnaire (eHLQ), to assess health literacy, such as the patient's motivation and ability to engage with digital technologies [24, 25]. A detailed summary of the content of each questionnaire is provided in the 'Supplementary Methods.'

Individual item scores were based on the Likert scales: 1-4 for both heiQ and eHLQ, and 1-6 for SUTAQ. For all scales, high values indicate positive health-related outcomes, except for the 'emotional distress' item in heiQ, where higher values mean greater emotional distress, and for 'privacy and discomfort' and 'care personnel concerns' in SUTAQ, for which a high score reflects a high level of concern. Descriptive statistical analyses were used to report the results from the questionnaires.

All analyses were carried out using Stata, version 14 [26]. Results from the questionnaire are presented as mean scores.

\section{Results}

A total of 56 patients were invited to participate in the study. Of those 21 did not reply to the invitation and 21 declined to participate. Of the 14 patients who took part (seven male and seven female), nine had RA, four had PsA, and one had AS. Other stakeholders included two doctors, two nurses, one medical secretary, and four other public servants involved in the management of the selected rheumatic diseases.

\subsection{Concept Mapping}

CM was applied to three focus group workshops: two groups with four patients with RA in each, and one group with four PsA patients. Patients had a mean age of 65.3 years (min-max: 49-76) and a mean disease duration of 10.8 years $(7-23)$.

During the CM workshops, 121 patient statements were generated and analyzed using cluster analyses; four key concepts emerged regarding e-Device implementation, which were reviewed by all workshop participants: technical usability, physical design, concerns, and enthusiasm. The four concepts (clusters), sub-clusters, and examples of statements that fell into each cluster/sub-cluster, are shown in Table 1. Homogenous response, also known as 'saturation,' was achieved; this indicated that the number of patients included was sufficient and so the option to expand the number of CM workshops was not used.

Each participant also rated the statement's relative importance with respect to patients receiving treatment using the e-Device. Participants rated 'easy to use,' 'the main function is to take the medication' and 'must be easy to handle when you have sore fingers,' as being the most important statements (Supplementary Figure 2; see the ESM). In contrast, 'it's hard to imagine how the needle is positioned in the device,' 'sharing information with the doctor can feel like being monitored,' and 'must be capable of handling flexible doses' were rated as being of relatively minor importance (Supplementary Figure 2). 
Table 1 Concepts produced from patient statements regarding the e-Device in the concept mapping workshops

\begin{tabular}{|c|c|c|}
\hline Concepts (clusters) & Sub-clusters & Patient statements \\
\hline Design and handling of the device & $\begin{array}{l}\text { Manageability (simple) } \\
\text { Size of the device } \\
\text { The needle }\end{array}$ & $\begin{array}{l}\text { Easy to use/simple set-up } \\
\text { Easier to handle than the syringe } \\
\text { Too clumsy/too big (should look more like a pen) } \\
\text { The needle should be thinner than it is now } \\
\text { An advantage that the needle is not visible }\end{array}$ \\
\hline $\begin{array}{l}\text { Technical features and additional } \\
\text { equipment }\end{array}$ & $\begin{array}{l}\text { Keep it simple } \\
\text { Software for wireless } \\
\text { communication } \\
\text { Setting of the device (flexibility } \\
\text { important) } \\
\text { Cooling system (when travelling) } \\
\text { Reminder, calendar, diary, etc. }\end{array}$ & $\begin{array}{l}\text { Main function: take the medication } \\
\text { Remote communication between patient and doctor/hospital } \\
\text { Flexibility } \\
\text { Communication with tablets and smart phones } \\
\text { Use the device for other type of medication (e.g., methotrexate) }\end{array}$ \\
\hline Concerns & $\begin{array}{l}\text { Will it be too much trouble? } \\
\text { The device is not for everybody } \\
\text { The device makes me dependent } \\
\text { Testing of the device } \\
\text { Data security }\end{array}$ & $\begin{array}{l}\text { Makes me independent/not flexible to the life I live } \\
\text { Too much control } \\
\text { I do not want to be controlled by the device } \\
\text { Difficult for persons unfamiliar with IT } \\
\text { Is there support, if needed urgently? } \\
\text { Has it been tested on patients? (The device/needle is placed } \\
\text { differently on the skin) } \\
\text { Sharing of data needs to be voluntary }\end{array}$ \\
\hline Enthusiasm & $\begin{array}{l}\text { Very useful } \\
\text { Safety }\end{array}$ & $\begin{array}{l}\text { Very helpful for those who do not like needles } \\
\text { Helpful for people with finger problems } \\
\text { It can easily be used as it is now } \\
\text { I do not have to worry about getting all the medication }\end{array}$ \\
\hline
\end{tabular}

$e$-Device electromechanical device

\subsection{Participatory Design}

Three male and one female patient (two with RA and two with PsA; mean age: 66.8 years; min-max: 54-76) who had participated in the CM workshops were recruited to individually participate in three iterative PD sessions each. The patients' brief was to use the three PD sessions to create a prototype that they felt would be an ideal e-Device that met their individual needs, whilst also bearing in mind all the concepts identified in the $\mathrm{CM}$ workshops. A number of key design features of the e-Device were highlighted by patients as being important during the PD sessions, including the size and shape, sound, the screen, connectivity options, charging options, and portability. A summary of the PD session discussions, and the 3D rendered images of the final personalized prototypes developed by the designer after the final PD session, are provided in Table 2.

\subsection{Stakeholder Evaluation}

To reduce the impact of power dynamics [27], SE comprised two group interviews (one with HCPs, the other with patients) and four individual interviews. The HCP group interview was conducted with two physicians, two nurses and one medical secretary from the department of rheumatology at the same hospital; the patient group interview was conducted with one male patient with RA and one female patient with AS. Solo interviews were conducted with the head of a clinical pharmacological department and member of RADS (the Danish council for the use of expensive hospital medicines), a chief nurse, and an economist (both the nurse and economist were at the management level in hospital rheumatology departments), and a regional medical and innovation consultant.

Analysis of the interview transcripts indicated two major themes: everyday life (with flexibility and functionality identified as sub-themes) and, relevance and clinical practice (with compliance and time as sub-themes). The drivers and barriers associated with the implementation and use of the standard route of injection [PFS and pre-filled pen (PFP)] and the e-Device, and how various elements could be adapted and developed in the current and next generation of the e-Device, were discussed (Table 3). In general, the results of the SE interviews highlighted broad 
Table 2 Central findings and prototypes produced from PD sessions with patients

Central findings from the PD sessions with the patient

Patient prototypes

Patient 1 (male, 54 years)

User does not like needles; the concept of 'ava ${ }^{\circledR}$, is well received

Ergonomic considerations; optional 3D-printed gripping area a possibility

Comments on screen angle; needs to be based on practical use. User puts screen flat on device for increased visibility while injecting. User would not inject in thigh-injecting into abdominal area seems 'easier' to the user

Tool reference; e-Device needs a quality feel—like a 'good' tool

Device must be identifiable as a medical device/tool

Patient 2 (male, 76 years)

No issues with needles-syringe is simple/ideal and is user controlled; doesn't like automated systems Existing device seems to require force in use due to bulky design

Wireless communication with clinic would be valuable

Ergonomic considerations

No need for a screen; haptic or audio-based feedback is enough

Use and design must be as simple as possible-device cannot complicate use

Patients must expect to be checked up on by the clinic-they receive expensive medication and proper use should be ensured

Novel loading concept created-resembling a bullet loading mechanism

Technology should be used to simplify things—not to complicate the users' life

Every patient has his own way of taking the medication

The sound of the 'ava ${ }^{\circledR}$, isn't good; the user feels like he is being stabbed upon motor-start

Cooling options for transport are discussed

User is 'happy to be of help to others'

If a riffle-like loading system is implemented, it is important that patients with gripping problems can use the system

The final product must 'speak' to the patients in the right way

Product needs to be self-diagnosing

Patient 3 (male, 66 years)

Syringe is ideal-if the needle is covered

Simplicity and ease of use are central points

User has no problems with the sharing of data with clinic

Flexible reminder system, text message, push or other solution suitable for a range of platforms

The 'ava ${ }^{\circledR}$, is 'overkill' (quote in relation to design). It is pleasant to hold, though

Design must be smaller, simpler and slimmer

All users have personal rituals for the use of their medication

Flexible splitting of screen and pen creates a range of user scenarios

Electrical toothbrush is used as an example of a 'buzzer' interface

Aesthetics are important-as is ease of use

Induction charging, the spilt elements and travelling/cooling options would all contribute to the flexibility of use

User referenced the sharing economy—travelling/cooling options could be shared by users and booked online

Vision impaired users and the advantages of non-visual feedback should be considered 
Table 2 continued

Central findings from the PD sessions with the patient

Patient prototypes

Patient 4 (female, 71 years)

The 'ava ${ }^{\circledR}$ ' is big, clumsy and heavy-the design makes this user 'feel worse'

Good if smartphone could be used for reminders

Syringe is an ideal solution

Design needs to be elegant- 'if I'm going to have to live with this-it needs to look good'

Very slim first prototype facilitates delicate grip

Active user-long-lasting cooling solution needed for travel; up to $72 \mathrm{~h}$ of off-grid use for overseas travelling

Customs and cargo handling of device should be considered

An active cooling solution could ease anxiety with the user when collecting medication

Hand impaired users should be considered

e-Device electromechanical device, $P D$ participatory design

differences in the types of perspectives shared by the different groups; patients were more focused on the functionality of the device and making suggestions relevant to incorporating the technology into everyday life, whilst key disease-management stakeholders were more focused on injection logging and the associated data compliance, timesaving considerations for patients and HCPs, the types of patients and treatment scenarios for which the e-Device would be relevant, and implementation of the e-Device in clinical practice.

\subsection{Patient-Reported Outcomes}

After participating in the evaluation on the e-Device, all 14 participating patients were invited to complete a questionnaire which included heiQ, SUTAQ and eHLQ to assess the participants' health literacy, the levels of empowerment they felt regarding the e-Device and their acceptance of technology in general. A total of seven male and five female patients $(86 \%)$ responded; demographic characteristics of the respondents are shown in Supplementary Table 1 (see the ESM). The results of the heiQ, SUTAQ, and eHLQ are shown in Supplementary Figure 3. While the patients who evaluated the e-Device and responded to the questionnaires had medium-high health literacy (based on feedback from the heiQ), the e-Device was associated with high levels of empowerment, and there were high levels of acceptability of e-Device technology (in general) among the group.

\section{Discussion}

The aim of this study was to assess the face validity and feasibility of the Parker Model at acquiring end-user perspectives on new self-injection device technologies. The Parker Model used a synergistic three-step qualitative method incorporating CM, PD, and SE, which was developed to inform the design, development, and implementation of a new e-Device for the subcutaneous selfadministration of CZP in patients with rheumatic disease. Unstructured feedback collected from all participants at the end of the study demonstrated that they thought the Parker model comprehensively captured all constraining concepts related to the device's design and use, and the model effectively generated feasible solutions to overcome all identified constraints; as such, the Parker Model method was regarded highly relevant for its purpose.

The results of the CM and PD phases of the study indicated that while the introduction of the e-Device for the disease management of chronic rheumatic diseases is a welcome development, no single e-Device design would be ideal for all patients_- 'no size fits all.' However, providing patients with a number of different injection device options to choose from would enable them to select an option suited to their needs. Overall, the Parker Model evaluation suggests that by carefully considering the feedback on the design, function, and implementation of the e-Device raised by patients and disease-management stakeholders, their expectations and requirements can be met through informed implementation and improvement of the e-Device.

The Parker Model methodology of using CM, PD, and SE has several advantages over using any one of these methods individually. Used together, these methods interact and support the flow of information from one session to the next, helping to mediate a co-creative process involving participants and investigators. The Parker method also has advantages over other qualitative research methods that are used in the development and implementation of medical devices. Ethnographic studies are field based analyses and so have the advantage of observing patients in a 'real world' setting [28]; however, they are associated with investigator interpretation bias [29], and they are resource intensive, limiting the number of participants and 
Table 3 Perspectives identified by SE of the applicability and relevance of introducing an e-Device to self-administer CZP for patients with rheumatic diseases

Key stakeholder perspectives and suggestions identified through SE interviews

\section{Functionality of the e-Device}

All stakeholders: the e-Device is ergonomic, fits well in the palm of the hand, easy to handle, yet relatively large. They found the dose dispenser cartridge easy to manage, though large when including the needle cap

Head nurse: device good for patients with arthritis; useful to have all equipment in one bag; relatively large bag is an issue for portability

HCPs: good display-letters are easy to read; offer the choice of different languages

Head nurse and patients: the reminder function is an advantage in the upstart phase

HCPs: concern that patients might find injection logging to be unwelcome monitoring

Patients: not concerned by injection logging since they already keep a record of their injections

HCPs: the non-visible needle is an advantage for patients afraid of needles

All stakeholders: other advantages are securing skin contact before an injection, automated injection, and choice of injection speed

Patients: satisfied with the standard route of CZP administration; the e-Device needs to offer more before they would consider changing

All stakeholders: why is the device for one medicine only? RADS member: it would be advantageous if the e-Device could be used to administer other medications, especially those with known poor compliance

Patients and the economist: unmet need is the dispenser cartridge having capacity for multiple doses. This would allow patients to take more doses at a time, reducing the frequency of hospital visits

Patient: no need for the device to tell you that the medication and the dose he is to administer are correct as only one medication and a particular dose can be administered with the device

Patient: relying on the e-Device would 'take away my empowerment'

Patient: access to the standard route of CZP administration is a necessary back-up in case of e-Device failure

Patient: battery power option would be convenient for portability and use while travelling

Head nurse: unmet need for patient information with FAQs, including who to contact if the device fails, and illustrations for patients who cannot read, and translation of the materials into Danish

\section{Flexibility}

Stakeholders: unmet need is flexibility in terms of the timing of injections. Most patients are experienced and capable of administering their medication on a schedule that best suits them. The patients stressed the importance of this flexibility to feel empowered in relation to their disease and treatment. A suggested solution was to set up the e-Device with slots of a few days for injections instead of fixed dates

HCPs: concerned that patients would need a hospital visit to adjust the device in case of a dose change, which would be time-consuming for both patients and HCPs. Suggestion: remote set-up of the e-Device, through an electronic connection between the e-Device and the hospital

Head nurse: patients might not remember to bring the e-Device to consultations when needed. Suggestion: incorporating a cooling bag for the medication into the storage case for the device

\section{Logging of data and compliance}

HCPs: the injection log may be helpful for patients who forget to take their medication, adding to treatment safety, and the option to use the injection $\log$ in consultations with the patient is an advantage

Economist: incorporating the injection log into the hospital databases would be an advantage for combining other information about the patient with their injection log, and for organizational and research purposes

RADS member: the injection log would be valuable for research and treatment decision-making (being able to compare compliance and treatment efficacy)

Patients: happy to share the injection log with HCPs because it would provide the HCP with the best information for decision-making; they are dependent on the HCP

\section{Time saving for patients and HCPs}

HCPs: the e-Device would be easy for patients to learn, but would not save time for nurses, who would need to instruct each patient on how to use the e-Device. More time may need to be spent by both the patient and the HCP if the patient forgets to bring the e-Device to consultations and additional consultations are required as a result. The potential need for a physician to be present during a nurse consultation in the case of a change in dose, this would result in extra time consumption for the physician

\section{Incorporating technology into everyday life}

HCPs: the e-Device should be able to communicate with the electronic systems already used in hospitals

Patients: the reminder system would only be useful if it was available on electronic devices that they use every day, not on the e-Device itself, which they turn on only to inject. The patients preferred the ability to connect to tablets and smartphones, over computers. They considered wireless connection to be modern, and connection by cable to be old-fashioned. Electronic communication between the home and hospital to transfer information would be valuable and would save on hospital visits

Head nurse: suggested a secure Danish website for sharing health information between the hospital and home; the option to remotely change the dose on the e-Device would be an advantage 
Table 3 continued

Key stakeholder perspectives and suggestions identified through SE interviews

For which patients and treatment scenarios will an e-Device be relevant?

All stakeholders: not relevant for patients happy with self-injection via the standard route, but the e-Device might be introduced as a choice to patients starting CZP treatment

Member of RADS: the e-Device is relevant for patients not able to self-inject via the standard route

Head nurse: the e-Device is relevant for compliant patients, those able to handle their medication from home, and patients who are familiar with electronics to some extent or are not afraid of it

Head nurse and economist: there is economic potential in switching patients who require either assistance with self-injection, or administration by infusion, to the e-Device; the member of RADS disagreed: switching patients receiving medication by infusion would not reduce contact time

\section{Implementation of an e-Device in clinical practice}

The economist, member of RADS, and HCPs: decisions regarding the choice of primary treatment prescribed to newly diagnosed patients are made at a national level; if the price of the e-Device was the same as for the standard route of CZP administration, then patient preferences and the injection log may be good arguments for the use of the e-Device

Member of RADS: advantages of the e-Device are not sufficient to recommend CZP as the primary treatment if more expensive than the alternative and the efficacy and adverse effect profiles are comparable

Member of RADS: a generic e-Device for the sole purpose of creating and injection log might save the healthcare system money because the injection log would allow non-compliant patients to be identified and would prevent those patients being switched to more expensive treatments

Head nurse: a concern is that the Danish accounting system is based on the number of hospital visits; implementing an e-Device would not be an incentive in a Danish hospital if it saves visits

The medical and innovation consultant: there are issues regarding data sharing, which requires approval by the health authorities, and the IT departments must be consulted early for the e-Device to be compliant with data security. Data management needs to be thoroughly described, including who owns the data, how will the data be shared, where and how will the data be stored, and who has access. If data are to be shared with and used in clinical practice, the data also need to be in a form compatible with the IT systems already in use

$C Z P$ certolizumab pegol, $e$-Device electromechanical device, $H C P$ healthcare professional, $R A D S$ the Danish council for the use of expensive hospital medicines, $S E$ stakeholder evaluation

generalizability of the data [28]. Semi-structured interviews provide the flexibility required for participants to give varied and personal responses, but also capture heterogeneous opinions that are difficult to generalize.

The Parker Model increased the generalizability of the $\mathrm{CM}$ results by using a focus-group format and a nominal group process [30], which gave each participant the opportunity to provide input (preventing more outspoken participants from dominating the discussion). This, and the $\mathrm{CM}$ process itself, ensured a variety of perspectives, while also reducing the number of clustered statements because participants were asked to identify the key concepts together [31]. The Parker Model further increased generalizability by ensuring that all participants involved in the PD session had also participated in the $\mathrm{CM}$ session. This helped to ensure that the prototypes developed during this session incorporated the feedback from all patients. The SE broadened the focus to include stakeholder input on the context of the e-Device and future iterations of it in the current healthcare setting, to evaluate device eligibility for implementation and how to make it readily beneficial in the public setting. Practically, since the three steps of the Parker Model can be applied in parallel, it is also an efficient way of capturing the various inputs from patients and other stakeholders.
High levels of treatment adherence among multiple sclerosis patients have been reported in studies examining the use of electronic injection devices that are similar to the e-Device evaluated in the current study; for example, allowing patients to individually adjust settings, such as injection speed and depth, and having the ability to electronically store data such as date and time of injections [32-34]. Consistent with these studies in multiple sclerosis, patients with rheumatic diseases recruited to provide their input on the e-Device in the current study reported high levels of e-Device acceptability and high levels of empowerment in the heiQ, SUTAQ, and eHLQ questionnaire. Patient engagement in healthcare is associated with better treatment adherence, which has the potential to improve health outcomes and patient care, and reduce treatment costs $[35,36]$. Patients' desire for empowerment was previously reported in an ethnographic study exploring contrasts in patient- and physician-reported views on living with RA [37], which, together with the results from the current study, suggests that ava ${ }^{\circledR}$ has the potential to improve patient engagement, which may improve treatment adherence.

The main limitations of the current study were associated with the relatively small number of patients and disease-management stakeholders, who were all from the 
same healthcare setting; stakeholder input from various institutions would have furthered the generalizability of the results. The small number of patients limited the range of ages that could be surveyed, and the patients were not all being treated for the same rheumatic disease, which may have introduced heterogeneity in the results. The male-tofemale ratio among the recruited patients was equal, which is not representative of the fact that some rheumatic diseases affect more females than males. The patients also had established disease, meaning that they were already accustomed to, and satisfied with, self-injection using the PFS or PFP, and had sufficient dexterity and hand function to operate the e-Device. No patients had to be excluded from the study due to being unable to use the device; however, the patients recruited in this study may not be representative of all patients with rheumatic disease, and no one device design is likely to be 'ideal' for all patients.

\section{Conclusions}

Overall, this study has demonstrated that the Parker Model is an effective qualitative method for collecting and generalizing responses from patients and other key stakeholders involved in disease management. This is the first time a composite, qualitative research model has been applied when introducing a new device to support the treatment of rheumatic diseases, and these resources can help ensure added value when developing devices for disease management. Although this study only evaluated the Parker Model in the context of a self-injection device, with further testing the model could also be applied to evaluate other medical devices, app technologies, and communication strategies; as such, the Parker Model has the potential to play a pivotal role in the future of personalized and outcome-based disease management models in a range of chronic disease areas.

Data Availability Statement Most of the data generated and analyzed during this study are included in this published article (and its supplementary information files). Some data generated and analyzed during the current study are not publicly available due to confidentiality clauses signed with patients and HCPs, but are available from the corresponding author on reasonable request.

\footnotetext{
Acknowledgements The authors thank the patients, the investigators, and their teams who took part in this study. The authors also acknowledge Debbie Nixon, DPhil, UCB Pharma, UK, for publication coordination and Hinal Tanna, PhD, and Simon Foulcer, PhD, from Costello Medical Consulting, UK, for medical writing and editorial assistance in preparing this manuscript for publication, based on the authors' input and direction.
}

Author Contributions The authors' contributions to this work are as follows: Substantial contributions to study conception/design or acquisition/analysis/interpretation of data: TSJ, MS, PCT, HCA, AL, LK, LS, IM, HG, and LEK. Drafting of the publication or revising it critically for important intellectual content: TSJ, MS, PCT, HCA, AL, LK, LS, IM, HG, and LEK. Final approval of the publication: TSJ, MS, PCT, HCA, AL, LK, LS, IM, HG, and LEK.

\section{Compliance with Ethical Standards}

Funding UCB Pharma and the Oak Foundation sponsored the study and the development of the manuscript; UCB Pharma reviewed the text to ensure that from the UCB Pharma perspective, the data presented in the publication are scientifically, technically, and medically supportable, that they do not contain any information that has the potential to damage the intellectual property of $\mathrm{UCB}$, and that the publication complies with applicable laws, regulations, guidelines, and good industry practice. The authors approved the final version to be published after critically revising the manuscript for important intellectual content.

Conflict of interest $\mathrm{T}$ Jørgensen has received speakers bureau honoraria from Abbvie, Biogen, Novartis, Roche, and UCB Pharma. H.C. Asmussen is a consultant for The Parker Institute and UCB Pharma. L.E. Kristensen has received speakers bureau honoraria from AbbVie, Amgen, BMS, Celgene, Eli Lilly, Janssen Pharmaceuticals, MSD, Novartis, Pfizer, and UCB Pharma. I. Mountian is an employee of UCB Pharma. P.C. Taylor has received research grants from UCB Pharma, Janssen, Galapagos, Eli Lilly, and Abide Therapeutics, and consulting fees from AbbVie, Eli Lilly, UCB Pharma, Pfizer, Biogen, Janssen, Sanofi, and GlaxoSmithKline. L. Klokker, M. Skougaard, A. Lee, L. Svejstrup, and H. Gudbergsen declare that they have no conflict of interest.

Ethical approval All procedures followed were in accordance with the ethical standards of the responsible committee on human experimentation (institutional and national) and with the Helsinki Declaration of 1964, as revised in 2013.

Informed consent Informed consent was obtained from all patients for their inclusion in the study.

Open Access This article is distributed under the terms of the Creative Commons Attribution-NonCommercial 4.0 International License (http://creativecommons.org/licenses/by-nc/4.0/), which permits any noncommercial use, distribution, and reproduction in any medium, provided you give appropriate credit to the original author(s) and the source, provide a link to the Creative Commons license, and indicate if changes were made.

\section{References}

1. Ford AC, Sandborn WJ, Khan KJ, Hanauer SB, Talley NJ, Moayyedi P. Efficacy of biological therapies in inflammatory bowel disease: systematic review and meta-analysis. Am J Gastroenterol. 2011;106(4):644-59 (quiz 60).

2. Singh JA, Saag KG, Bridges SL Jr, et al. 2015 American College of Rheumatology Guideline for the treatment of rheumatoid arthritis. Arthritis Rheumatol. 2016;68(1):1-26.

3. Tabas I, Glass CK. Anti-inflammatory therapy in chronic disease: challenges and opportunities. Science. 2013;339(6116):166-72. 
4. Bolge SC, Goren A, Tandon N. Reasons for discontinuation of subcutaneous biologic therapy in the treatment of rheumatoid arthritis: a patient perspective. Patient Prefer Adherence. 2015;9:121-31.

5. Borrás-Blasco J, Gracia-Pérez A, Casterá MD-E, Rosique-Robles JD, Abad J. Educational session as a tool to increase patient satisfaction of switching etanercept from the prefilled syringe to the autoinjection pen. Expert Opin Biol Ther. 2013;13(8):1103-8.

6. Zimmer PA, Braun LT, Fraser R, Hecht L, Kelliher F. Promoting success in self-injection: listening to patients. Medsurg Nurs Off J Acad Med Surg Nurses. 2015;24(4):279-82.

7. Yeaw J, Benner JS, Walt JG, Sian S, Smith DB. Comparing adherence and persistence across 6 chronic medication classes. J Manag Care Pharm. 2009;15(9):728-40.

8. Marengo MF, Suarez-Almazor ME. Improving treatment adherence in patients with rheumatoid arthritis: what are the options? Int J Clin Rheumtol. 2015;10(5):345-56.

9. Markiewicz K, van Til JA, IJzerman MJ. Medical devices early assessment methods: systematic literature review. Int $\mathrm{J}$ Technol Assess Health Care. 2014;30(2):137-46.

10. EMA. Cimzia: Summary of Product Characteristics Secondary EMA. Cimzia: Summary of Product Characteristics 2017. http:// www.ema.europa.eu/ema/index.jsp?curl=pages/medicines $/$ hum an/medicines/001037/human_med_001294.jsp\&mid=WC0b01ac $058001 \mathrm{~d} 124$.

11. Trochim W, Kane M. Concept mapping: an introduction to structured conceptualization in health care. Int J Qual Health Care. 2005;17(3):187-91.

12. The Concept System ${ }^{\circledR}$ Global MAX $^{\mathrm{TM}}$ (Build 2016.046.12) [Web-based Platform]. Ithaca; 2016. http://www. conceptsystemsglobal.com.

13. Trochim WM, Cook JA, Setze RJ. Using concept mapping to develop a conceptual framework of staff's views of a supported employment program for individuals with severe mental illness. J Consult Clin Psychol. 1994;62(4):766-75.

14. Trochim WM, Linton R. Conceptualization for planning and evaluation. Eval Program Plann. 1986;9(4):289-308.

15. Crabtree BF, Miller WL. Using codes and code manuals-a template organizing style of interpretation. In: Crabtree BF, Miller WL, editors. Doing qualitative research. 2nd ed. Thousand Oaks: SAGE Publications Inc; 1999. p. 163-77.

16. Hansen MB, Vedung E. Theory-based stakeholder evaluation. Am J Eval. 2010;31(3):295-313.

17. Braun V, Clarke V. What can "thematic analysis" offer health and wellbeing researchers? Int J Qual Stud Health Well Being. 2014;9:26152.

18. Guest G, MacQueen KM, Namey EE. Applied thematic analysis. California: Sage; 2011.

19. Osborne RH, Elsworth GR, Whitfield K. The Health Education Impact Questionnaire (heiQ): an outcomes and evaluation measure for patient education and self-management interventions for people with chronic conditions. Patient Educ Couns. 2007;66(2):192-201.

20. Osborne RH, Batterham R, Livingston J. The evaluation of chronic disease self-management support across settings: the international experience of the health education impact questionnaire quality monitoring system. Nurs Clin $\mathrm{N}$ Am. 2011;46(3):255-70.
21. Epstein J, Osborne RH, Elsworth GR, Beaton DE, Guillemin F. Cross-cultural adaptation of the Health Education Impact Questionnaire: experimental study showed expert committee, not back-translation, added value. $\mathrm{J}$ Clin Epidemiol. 2015;68(4):360-9.

22. Elsworth GR, Nolte S, Osborne RH. Factor structure and measurement invariance of the Health Education Impact Questionnaire: does the subjectivity of the response perspective threaten the contextual validity of inferences? SAGE Open Med. 2015;3:2050312115585041.

23. Hirani SP, Rixon L, Beynon M, et al. Quantifying beliefs regarding telehealth: development of the Whole Systems Demonstrator Service User Technology Acceptability Questionnaire. J Telemed Telecare. 2017;23(4):460-9.

24. Osborne RH, Batterham RW, Elsworth GR, Hawkins M, Buchbinder R. The grounded psychometric development and initial validation of the Health Literacy Questionnaire (HLQ). BMC Public Health. 2013;13:658.

25. Maindal HT, Kayser L, Norgaard O, Bo A, Elsworth GR, Osborne RH. Cultural adaptation and validation of the Health Literacy Questionnaire (HLQ): robust nine-dimension Danish language confirmatory factor model. Springerplus. 2016;5(1):1232.

26. StataCorp. Stata statistical software: release 14. College Station: StataCorp LP; 2015.

27. Smithson J. Using and analysing focus groups: limitations and possibilities. Int J Soc Res Methodol. 2000;3(2):103-19.

28. Goodson L, Vassar M. An overview of ethnography in healthcare and medical education research. J Educ Eval Health Prof. 2011;8:4.

29. Morgan-Trimmer S, Wood F. Ethnographic methods for process evaluations of complex health behaviour interventions. Trials. 2016;17(1):232.

30. Van de Ven AH, Delbecq AL. The nominal group as a research instrument for exploratory health studies. Am J Public Health. 1972;62(3):337-42.

31. Leung FH, Savithiri R. Spotlight on focus groups. Can Fam Physician. 2009;55(2):218-9.

32. Krol M, de Voer G, Osowski U. Patient adherence to subcutaneous IFN beta-1a injections using the RebiSmart( $\left({ }^{\circledR}\right)$ injection device: a retrospective real-world study among Dutch and German patients with multiple sclerosis. Patient Preference Adherence. 2017;11:1189-96.

33. Willis H, Webster J, Larkin AM, Parkes L. An observational, retrospective, UK and Ireland audit of patient adherence to subcutaneous interferon beta-1a injections using the RebiSmart( $\left({ }^{-}\right)$ injection device. Patient Preference Adherence. 2014;8:843-51.

34. Devonshire VA, Feinstein A, Moriarty P. Adherence to interferon $\beta-1$ a therapy using an electronic self-injector in multiple sclerosis: a multicentre, single-arm, observational, phase IV study. BMC Res Notes. 2016;9:148.

35. Berwick DM, Nolan TW, Whittington J. The triple aim: care, health, and cost. Health Aff (Project Hope). 2008;27(3):759-69.

36. Hibbard JH, Cunningham PJ. How engaged are consumers in their health and health care, and why does it matter? Res Brief. 2008;8:1-9.

37. Fair BS. Contrasts in patients' and providers' explanations of rheumatoid arthritis. J Nurs Scholarsh. 2003;35(4):339-44. 